\title{
ECRH assisted plasma start-up with toroidally inclined launch: multi-machine comparison and perspectives for ITER
}

\author{
J. Stober ${ }^{1}$, G.L. Jackson ${ }^{2}$, E. Ascasibar ${ }^{3}$, Y.-S. Bae ${ }^{4}$, J. Bucalossi ${ }^{5}$, \\ A. Cappa $^{3}$, T. Casper $^{6}$, M.-H. Cho ${ }^{7}$, Y. Gribov ${ }^{6}$, G. Granucci ${ }^{8}$, \\ K. Hanada ${ }^{9}$, J. Hobirk ${ }^{1}$, A.W. Hyatt ${ }^{2}$, S. Ide ${ }^{10}$, J.-H. Jeong ${ }^{7}$, M. Joung ${ }^{4}$, \\ T. Luce $^{2}$, T. Lunt ${ }^{1}$, W. Namkung ${ }^{7}$, S.-I. Park ${ }^{7}$, P.A. Politzer ${ }^{2}$, \\ J. Schweinzer ${ }^{1}$, A.C.C. Sips ${ }^{11}$, the ASDEX Upgrade team ${ }^{1}$, the TJ-II \\ team $^{3}$ and the ITPA "Integrated Operations Scenarios" group members \\ and experts \\ ${ }^{1}$ Max-Planck-Institut für Plasmaphysik, EURATOM-Association, Garching, Germany \\ ${ }^{2}$ General Atomics, PO Box 85608, San Diego, CA 92186-5608, USA \\ ${ }^{3}$ Laboratorio Nacional de Fusión, Asociación EURATOM/CIEMAT, 28040, Madrid, Spain \\ ${ }^{4}$ National Fusion Research Institute, Gwahangno 113, Yuseong-gu, Daejeon 305-333, Korea \\ ${ }^{5}$ Association Euratom-CEA, Cadarache, F-13108 St. Paul-lez-Durance, France \\ ${ }^{6}$ ITER Organisation, Route de Vinon sur Verdon, F-13115 St. Paul-lez-Durance, France \\ ${ }^{7}$ Pohang University of Science and Technology, San 31, Hyoja-dong, Nam-gu, Pohang \\ 790-784, Korea \\ ${ }^{8}$ Associazione Euratom-ENEA sulla Fusione, IFP-CNR, Via R. Cozzi 53, 20125 Milano, \\ Italy \\ ${ }^{9}$ RIAM, Kyushu University, RIAM, Kasuga, Fukuoka, 816-8580 Japan \\ ${ }^{10}$ Japan Atomic Energy Agency, 801-1, Mukouyama, Naka, Ibaraki-ken, 311-0193 Japan \\ ${ }^{11}$ EFDA JET, Culham Science Centre, Abingdon, OX14 3DB, UK \\ e-mail: Joerg.Stober@ipp.mpg.de
}

\begin{abstract}
ECRH assisted plasma break down is foreseen with full and half magnetic field in ITER. As reported earlier, the corresponding O1- and X2-schemes have been successfully used for pre-ionisation and breakdown assist in present day devices. This contribution reports on common experiments studying the effect of toroidal inclination of the ECR beam, which is $\geq 20^{\circ}$ in ITER. All devices could demonstrate successful breakdown assist also for this case, although in some experiments the necessary power was almost a factor of two higher compared to perpendicular launch. Differences between the devices with regard to the required power and vertical field are discussed and analysed. In contrast to most of these experiments, ITER will build up loop voltage prior to the formation of the field null due to the strong shielding by the vessel. Possible consequences of this difference are discussed.
\end{abstract}

\section{Introduction}

The standard procedure to start a Tokamak plasma (ohmic start-up) relies on plasma breakdown in the presence of a toroidal electrical field $\vec{E}_{\Phi}$. According to Townsend's theory 
this is facilitated by the optimisation of the neutral gas pressure (usually adjusted by the so-called pre-fill) and the maximum connection length of the magnetic field lines to the vessel wall, the latter corresponding to the distance between electrodes in Townsend's theory. Additionally, $\vec{E}_{\Phi}$ drives a toroidal ohmic current in the initial plasma. This current is an essential component of the evolving MHD equilibrium of the Tokamak. The formation of this equilibrium increases thermal insulation such that the ohmic heating is sufficient to generate a fully ionised plasma of several hundred $\mathrm{eV}$ electron temperature. On ITER, the superconducting solenoid and the thick vessel walls limit the toroidal electrical field available for plasma breakdown well below the values used in most of the operating Tokamaks. Since ITER is significantly larger than these Tokamaks one might argue that the connection length is larger and breakdown should be possible at lower electrical field. Such an argument neglects the quality of the field null which is not easy to predict, especially in the presence of vessel shielding and eddy currents. Data from existing machines at least do not show a clear size scaling of the electrical field necessary for ohmic breakdown (see table 1 below). This rose concern if the foreseen electrical field in ITER will allow reliable breakdown. In order to come to a conservative prediction on the breakdown behaviour in ITER, in this study machines are compared on the basis of the (measured) electrical fields, neglecting possible variations in (estimated) connection lengths.

Although experiments on JET [1], DIII-D [2] and Tore Supra (TS) [3] show that ohmic plasma start-up with the electrical field value of ITER $(0.3 \mathrm{~V} / \mathrm{m})$ is marginally possible for optimised magnetic configuration, assist of plasma breakdown by electron cyclotron resonance heating ECRH is highly desirable for ITER to increase the operational margin. The relevance of ECRH assist is highlighted by the first experiments on KSTAR in 2008: ECRH assist was necessary to break down the first plasmas [4]. This access route to plasma formation was successively optimised and in 2010 even purely ohmic breakdown was achieved [5]. To achieve initial plasma breakdown, ECRH can either create a low temperature plasma prior to the application of a loop voltage or in the presence of a loop voltage it may relax the Paschen-Criterium by additional acceleration of an otherwise sub-critical electron distribution [6]. In the subsequent current formation phase, when closed flux surfaces form and the electron temperature rises, ECR heating of the electrons can facilitate the sustainment of the breakdown through the radiation barrier when light impurities are ionized and radiate a significant fraction of the ohmic heating power. ECRH assist has already been investigated previously by the ITPA Steady-State-Operation group [1] and satisfactory results have been obtained for the fundamental and 2nd harmonic resonances. Both resonances may be used in ITER when operating with full and half magnetic field respectively to match the gyrotron frequency of $170 \mathrm{GHz}$. As explained below the optimum polarisation is O-mode in case of the fundamental resonance $(\mathrm{O} 1)$ and X-mode for the second harmonic (X2). Most of these results were performed with ECRH launched perpendicular to the magnetic field, in contrast to the planned situation in ITER where the minimum toroidal injection angle is $20^{\circ}$ [7]. As discussed in detail below, the initial results with toroidally inclined launch were contradictory. To clarify the effect on toroidal inclination, the new ITPA Integrated-Operational-Scenarios group initiated a continuation of the joint experiments with the participation of the Tokamaks 
AUG(X2), DIII-D(X2), FTU(O1), KSTAR(X2), Tore Supra (O1, X2), and of the Stellarator TJ-II (X2). It should be noted that during the initial collision-less heating-phase of the preionisation sequence, electron acceleration due to ECRH is dominantly perpendicular to the magnetic field [8], such that the large scale toroidal geometry does not play a significant role in this phase as long as no loop voltage is applied. Since in this study in all Tokamaks except FTU and TS the plasma was ionized by ECRH before the onset of the loop voltage, it is sensible to include as well Stellarators into a comparison of the conditions for the collisionless heating phase. In general it is noted that plasma breakdown using ECRH is the standard method in Stellarators and no principal problems with toroidally inclined beams have been reported. A systematic study on breakdown using mainly the $2^{\text {nd }}$ harmonic resonance in Heliotron $\mathbf{J}$ is presented in [9].

The rest of the paper is structured as follows: in the next section the effect of the toroidal beam inclination is described and analysed, which was the basic motivation for this study. During the study other parameters turned out to be important which are discussed separately in subsequent sections, i.e. poloidal launching angle, prefill pressure, and field null structure. Apart from the initial avalanche process also the current formation and impurity ionisation are significantly influenced by ECRH, which is discussed in a separate section. This is followed by a discussion of the consequences of these findings for ITER also addressing minimum power requirements and the adaption to the breakdown scheme foreseen in ITER, which differs from most of the experiments described here due to the strong vessel screening of magnetic flux modifications in ITER.

It is beyond the scope of this work to model the current formation phase. As outlined in section 6 , the evolution during this phase depends crucially on poorly controlled quantities such as induced vessel currents and impurity sources. The successive phase of plasma-current ramp-up is modeled by several groups as reported in $[10,11,12,13]$.

\section{ECRH breakdown assist with toroidally inclined ECRH}

AUG, DIII-D [14], KSTAR, and TJ-II find that ECRH assisted breakdown in X2-mode is possible with a toroidal injection angle of $20^{\circ}$ (or larger). FTU [15] obtains the same results for O1 in line with the older results from TS and JT-60U. These results indicate that a toroidal inclination of $20^{\circ}$ as foreseen for ITER does in general not pose additional complications for plasma breakdown with ECRH, although some parameters need readjustment such as prefill and ECRH power.

The results of the individual machines are detailed in table 1 for $\mathrm{O} 1$ and X2 heating. In all Tokamaks it was possible to reduce the necessary loop voltage by using ECRH assist. In most cases the toroidal $\vec{E}$-field on axis $\left(\vec{E}_{\Phi}\right)$ remained below the foreseen ITER value of $0.3 \mathrm{~V} / \mathrm{m}$. Exceptions are $\mathrm{O} 1$ experiments at FTU and X2 experiments at TS. FTU did not try lower voltage and in TS a non-optimised poloidal field null is regarded as reason for the unusual behavior.

As already reported in [1], a significant difference usually found between the O1- and X2schemes is the time delay between the start of the ECRH and a measurable plasma ionization 


\begin{tabular}{|c|c|c|c|c|c|c|c|c|c|c|c|c|}
\hline \multirow[b]{2}{*}{ Device } & Ohmic & \multicolumn{2}{|c|}{ O1 perp } & \multicolumn{3}{|c|}{ O1 inclined } & \multicolumn{2}{|c|}{$\mathbf{X} 2$ perp } & \multicolumn{3}{|c|}{$\mathbf{X 2}$ inclined } & \multirow[b]{2}{*}{ frequency } \\
\hline & $\vec{E}_{\min }$ & $\vec{E}_{\min }$ & $P_{E C R H}$ & $\vec{E}_{\text {min }}$ & $P_{E C}$ & angle & $\vec{E}_{\min }$ & $P_{E C R H}$ & $\vec{E}_{\min }$ & $P_{E C R H}$ & angle & \\
\hline AUG & 0.52 & 0.3 & 0.4 & & & & 0.2 & 1.0 & 0.2 & 1.6 & 20 & $\begin{array}{l}\mathrm{O} 1: 105, \\
\mathrm{X} 2: 105,140\end{array}$ \\
\hline DIII-D & $\begin{array}{l}0.25[2] \\
0.42[14\end{array}$ & $0.15[2$ & 0.8 & & & & $0.21[10]$ & 1.2 & $0.3[14$ & 0.6 & 24 & $\begin{array}{l}\mathrm{O} 1: 60, \\
\mathrm{X} 2: 60,110\end{array}$ \\
\hline FTU & 1.3 & 0.41 & 0.8 & 0.69 & 0.4 & 20 & & & & & & $140[15]$ \\
\hline JT-60U & 1.5 & & & 0.26 & 0.4 & 20 & & & & & & $110[16]$ \\
\hline KSTAR & $0.38[5]$ & & & & & & 0.24 & 0.35 & 0.24 & 0.35 & 15 & $84[4]$ \\
\hline & & & & & & & 0.29 & 0.24 & 0.29 & 0.24 & 20 & 110 [17] \\
\hline TS & 0.3 & 0.15 & 0.25 & 0.25 & 0.3 & 30 & 0.5 & 0.5 & 0.6 & 0.5 & 30 & $118[3,18]$ \\
\hline $\mathrm{T}-10$ & 0.44 & & & & & & & & 0.28 & 0.45 & 21 & 140 [19] \\
\hline QUEST & & $>3.0$ & 0.06 & & & & & & & & & 8.2 \\
\hline TJ-II & - & & & & & & - & 0.08 & - & 0.14 & $>30$ & 53.2 \\
\hline
\end{tabular}

Table 1. Optimized breakdown parameters $\left(\vec{E}\right.$ in $\mathrm{V} / \mathrm{m}, P_{E C R H}$ in $\mathrm{MW}$, angle in degree, frequency in $\mathrm{GHz}$ ). $\vec{E}_{\min }$ corresponds to the minimum in a sequence of $\vec{E}_{\Phi}$ values, which are themselves the maximum values of $\vec{E}_{\Phi}$-time-traces observed in separate discharges of the respective study. $\mathrm{P}_{E C R H}$ is the minimum power successfully tested with the cited value of $\vec{E}_{\min }$. As discussed in the text below, these numbers are not the result of a multi-dimensional minimisation procedure, but rather singular points found in usually one-dimensional scans. Especially the loop voltages at inclined angles in AUG, DIII-D and FTU were not minimised. For a single device, the field null structure may vary between different entries (except KSTAR (84 GHz) and TJ-II). In ITER $\vec{E}_{\Phi}$ cannot be larger than $0.3 \mathrm{~V} / \mathrm{m}$ and the toroidal launching angle of the ECRH beams is $>20^{\circ}$ degrees.

(usually determined by $H_{\alpha}$ emission). With $\mathrm{O} 1$ an increase of this emission can be measured immediately after ECRH is switched on, independent of injection angle. With X2 the increase is usually delayed by several ms (during which the full ECRH power has to be absorbed by in-vessel components) $\ddagger$. This delay is dependent on the inclination angle, as demonstrated in figure 1 for TJ-II. As in DIII-D, AUG, and TS, the delay increases with the inclination angle especially close to the ECRH power threshold. For KSTAR the situation is less clear. At least for the first ten degrees the delay is reduced [4].

To understand the difference between fundamental and 2 nd harmonic resonance, the preionisation process has to be described in some more detail: As an ECR-beam is injected into the neutral gas without a plasma, it is reflected, diffused, de-polarised and damped on in-vessel components and an undefined mixture of $\mathrm{O}$-and $\mathrm{X}$-mode polarisation will fill the whole vessel. The wave-field will still be highest in the injected beams, but also in other fractions of the vessel volume the intensity may be high depending on the injection angle, geometry of the first reflections, beam divergence and wall material. The latter determines the absorbed fraction per reflection. In [20] this fraction has been determined for $140 \mathrm{GHz}$

$\$$ In cases of ECRH power well above the threshold and optimised prefill pressure and field null the delay can be less than one ms for X2 in DIII-D. For the DIII-D case shown in fig. 1 of [14] the delay has been checked to be $0.9 \mathrm{~ms}$ between onset of ECRH and onset of $D_{\alpha}$. It is an open question if this should be regarded as similarly fast as the delay for $\mathrm{O} 1$ heating, which has been checked to be $0.5 \mathrm{~ms}$ for the so far only (and therefore not optimised) O1-case in AUG (see [1]). 


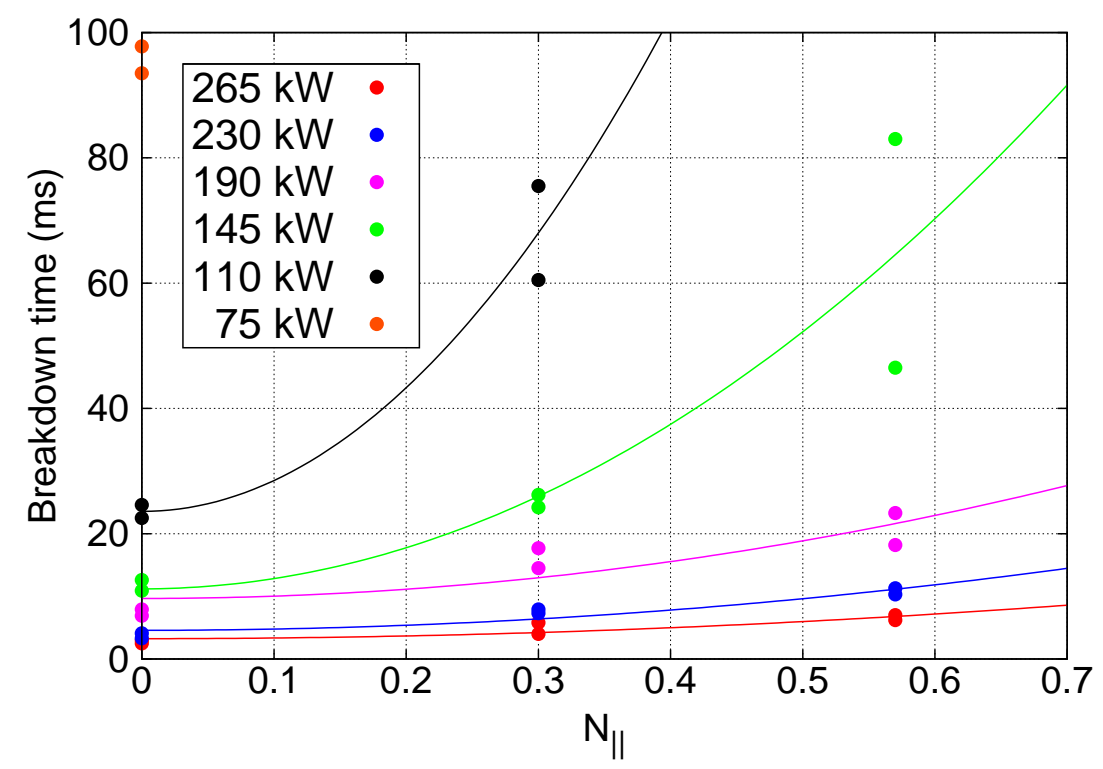

Figure 1. Effect of variation of the toroidal injection angle in TJ-II. Shown is the delay of the $H_{\alpha}$ rise after turning on the ECRH as function of $N_{\|}$for several ECRH powers (X2) with constant prefill of $5 \cdot 10^{-5}$ mbar $\mathrm{H}_{2}$ (\#23930-\#23965). $N_{\|}>0.55$ corresponds to a toroidal injection angle $>30^{\circ}$. $\left(N_{\|}\right.$is the refractive index $N$ multiplied with the cosine of the angle between wave vector and magnetic field. Here it refers to the plasma edge where $N \approx 1$.)

to be $\approx 1 / 20$ for graphite and $\approx 1 / 500$ for $\mathrm{W}$. A free electron will be accelerated perpendicular to the magnetic field since it dominantly absorbs on the $\mathrm{X}$-mode polarisation. (The reason to inject the fundamental wave as O-mode is an X-mode cut-off at the low field side, which develops at very low densities, such that O-mode is necessary to assist sustainment of the breakdown through the radiation barrier later in the discharge.) By non-ionising collisions with the neutral gas the electron gains parallel momentum and moves to other regions of the plasma volume, where the $\vec{E}$ field may be different. Since the magnetic field lines are not closed, it will finally be lost to the wall. The avalanche criterion basically is: do slow free electrons have in average the chance to accumulate so much ECRH power that they do create another (initially slow) electron by ionisation of a neutral atom before they hit the wall? The delay time which was observed corresponds in this picture to the time which is necessary to accelerate the first slow electrons to approximately $20 \mathrm{eV}$. For the fundamental resonance, absorption is very efficient even at low temperatures. Therefore acceleration is prompt. In contrast, $\mathrm{X} 2$ absorption vanishes for zero temperature and the heating rate is proportional to the electron perpendicular energy and the electric field. This leads to an exponential growth of the initially small $(0.03 \mathrm{eV})$ electron energy in time and the electric field determines the exponential growth time. This model has been quantitatively analysed for DIII-D experiments in [8] and good agreement with experiment was found. Due to the exponential dependence on the electrical field, electrons are accelerated dominantly in regions of highest ECR-wave field. Results from T-10 [19] on the effect of beam focusing point in the same direction, i.e. the delay is significantly increased if the beam is defocused, which strongly reduces the wave field. 


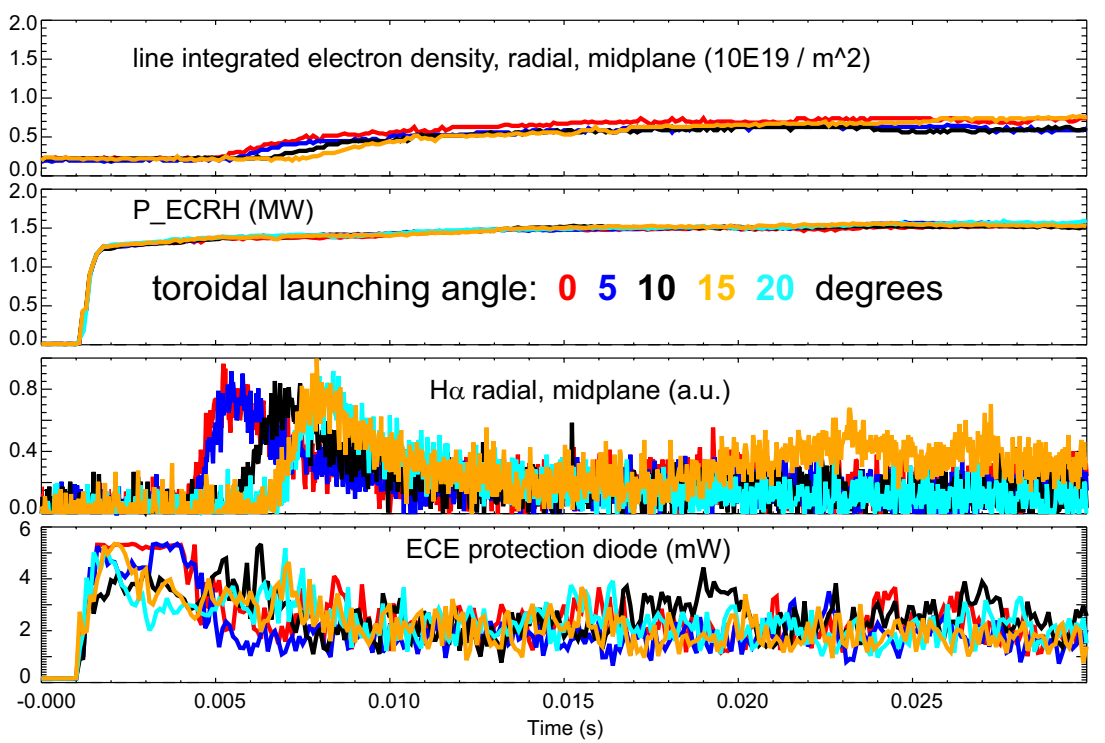

Figure 2. Scan of toroidal launching angle in $\mathrm{AUG}$ for $140 \mathrm{GHz} \mathrm{X} 2$-pre-ionisation for a toroidal magnetic field on axis of $2.3 T$ including effect on stray radiation. No significant differences were observed during the subsequent current formation phase, i.e. for times $>$ $0.03 \mathrm{sec}$ (not shown).

Potentially, the variation of the delay with beam inclination may also be related to a variation of the electrical field. For perpendicular launch, reflections keep the beam center in the poloidal plane of injection, in this region the field is enlarged and a significant toroidal decay is expected. In case of toroidally inclined injection the enhancement by multiple reflections is reduced as is the maximum field strength.

Stray-radiation detection in AUG supports this explanation as shown in fig. 2 for a toroidal angle scan of the ECR beam in otherwise identical discharges. The above mentioned delay of the $\mathrm{H}_{\alpha}$-rise is clearly seen. The bottom trace shows the stray power entering the ECE optics, which is $22^{\circ}$ degrees or $0.8 \mathrm{~m}$ toroidally separated from the next ECRH launcher which directs its beam (diameter during first path $<0.1 \mathrm{~m}$ ) successively away from the diode as the toroidal inclination is increased. The diode signal at the onset of the ECRH is saturated for normal inclination and decreases with increasing inclination. As the $\mathrm{H}_{\alpha}$ signal rises the stray radiation is reduced in all cases due to increased absorption on electrons with several $\mathrm{eV}$ of energy created by the avalanche process. Similar observations are made with sniffer probes in FTU. These results may suggest that strength of electrical field and the delay time are anti-correlated.

The much higher absorption of $\mathrm{X} 1$ compared to $\mathrm{X} 2$ also has the effect that for $\mathrm{X} 1$ the field strength of the initial stray field is strong enough to breakdown a plasma along the whole resonance cylinder (i.e. $B=\frac{m_{e} \omega}{n \cdot e}, n$ being the order of the harmonic), whereas for $\mathrm{X} 2$ this is only the case in the vicinity of the points where the initial beams cross the resonance cylinder, typically close to the midplane. This can be nicely observed with fast visible cameras in several devices. For fundamental resonance heating in TS fig. 3 shows that the resonance cylinder lights up within the timing uncertainty, as the ECRH is switched on. In contrast 

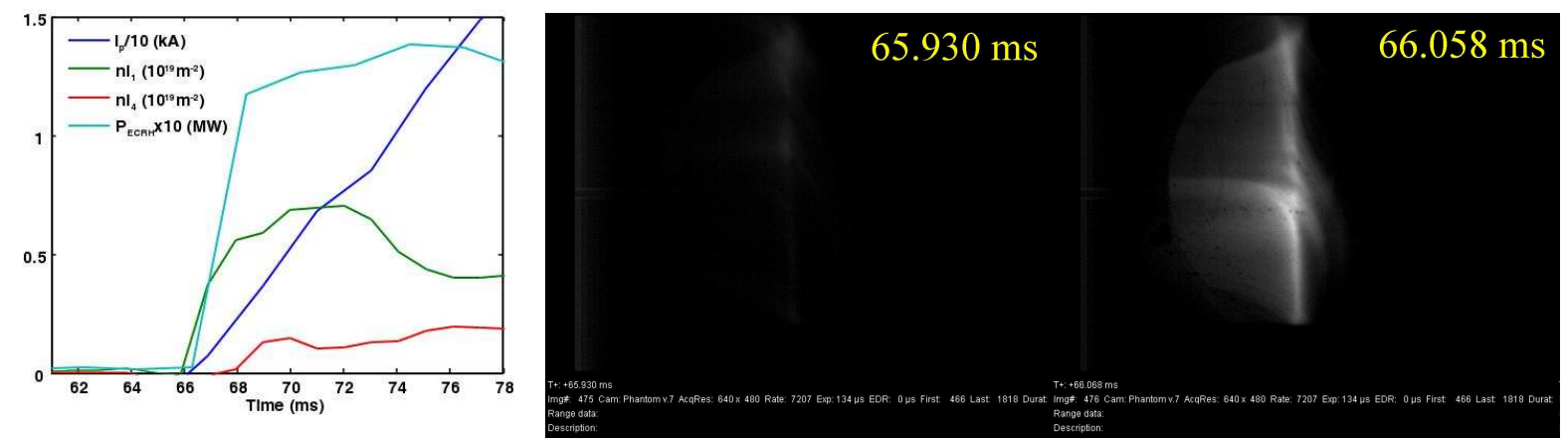

Figure 3. $\mathrm{O} 1$ pre-ionisation in TS (TS-42249): Time traces of $P_{E C R H}, I_{p}, \overline{n_{e}}$ (2 lines) and two frames from a CCD camera without filter, i.e. mostly $H_{\alpha}$ emission from [3]. Uncertainty of ECRH timing: $\pm 1 \mathrm{~ms}$.

fig. 4 shows that with $\mathrm{X} 2$ a plasma forms in the AUG midplane and extends upwards and downwards along the resonance with a vertical speed of 50-100 m/s. With $B_{z} / B_{\Phi} \approx 0.001$, the speed along field lines is $50-100 \mathrm{~km} / \mathrm{s}$, of the order of the generalised ion sound speed, which is $20 \mathrm{~km} / \mathrm{s}$ for $10 \mathrm{eV}$ electrons and cold ions (taking into account that in the expanding plasma the electrons have to drag their ions with them to maintain quasi-neutrality). §

According to this model of X2 heating the breakdown avalanche should start at the point of highest field on the resonance cylinder. This has clearly been observed in Heliotron J [9]. In Tokamaks a toroidal evolution has not yet been resolved in time. In contrary, in several of the Tokamak experiments of this study, it is found that the combination of several gyrotrons with beams crossing the resonance cylinder at the same $R, z$ but different $\Phi$ reduces the time between ECRH switch-on and the appearance of the $H_{\alpha}$ emission. The process is analysed in more detail in [8]: after an initial collision-less heating phase the accelerating electrons become collisional with respect to non-ionising collisions with the neutral gas, such that perpendicular energy is transfered to parallel energy. The electrons start to rotate toroidally following magnetic field lines. At that point every region along the magnetic field line where ECR-wave-field is high contributes to the further acceleration of the electron. Since $B_{z}$ usually is of the order 0.5-5 $\mathrm{mT}$ (see section 5) the vertical displacement per toroidal turn is of the order $0.2-2 \mathrm{~cm}(R=1.75 \mathrm{~m})$ or $0.8-8 \mathrm{~cm}(R=7 \mathrm{~m})$, such that even a single beam is passed several times. Therefore one expects that the plasma at the onset of the breakdown avalanche has a significant degree of toroidal symmetry. However, the fast camera measurements made in several Tokamaks using toroidal views are not really suited to characterise toroidal asymmetries, so the experimental evidence of a toroidally symmetric growth of the $H_{\alpha}$ emission in Tokamaks is still weak.

$\S$ Here and in the following the indicees $R, \Phi$ and $z$ represent the projections in the radial, toroidal and vertical direction. An index $p o l$ refers to the length of the vector projected into the $R, z$-plane. 

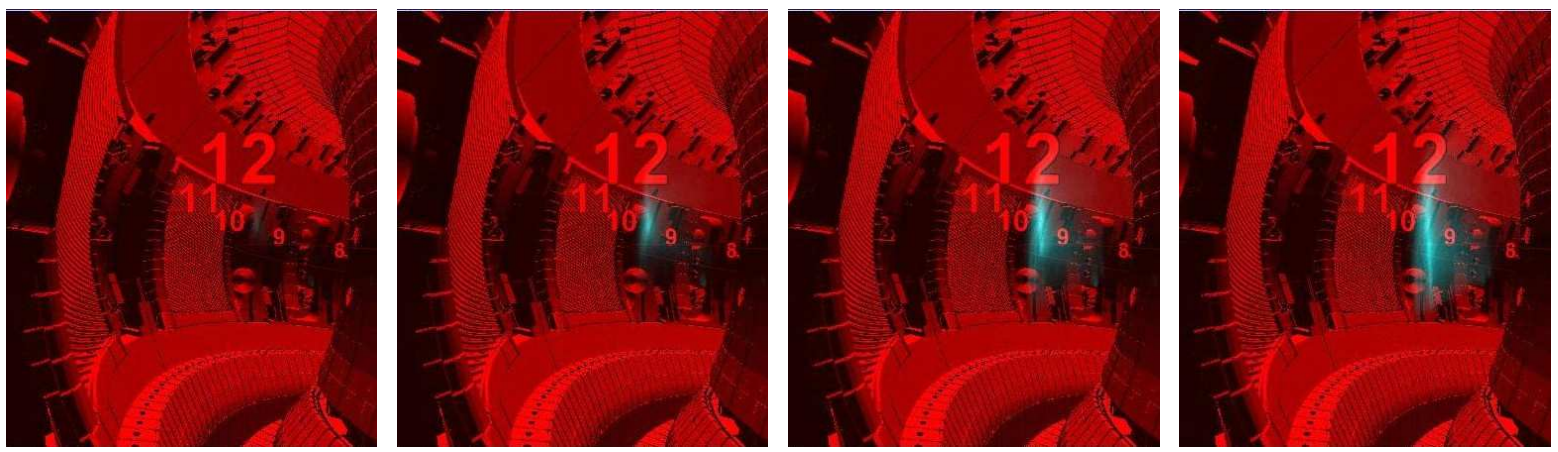

Figure 4. $\mathrm{X} 2$ pre-ionisation in AUG (\#23177): 4 frames from a CCD camera without filter, i.e. mostly $H_{\alpha}$ emission (light blue) in front of visualised CAD-data (red, numbers correspond to sectors) at $9.1 \mathrm{~ms}, 10.1 \mathrm{~ms}, 11.2 \mathrm{~ms}, 12.2 \mathrm{~ms}$. A total of 1.6 MW of ECRH was switched on (within $1 \mathrm{~ms}$ ) at $0.0 \mathrm{~ms}$, provided by four mid-plane launchers. At $12 \mathrm{~ms}$ the maximum of the $H_{\alpha}$ emission occurs. One launcher is visible in sector 8 but the intersection with the resonance cylinder is out of view. Therefore the light is probably emitted from a toroidal structure. Its height can be estimated by comparison to the height of the ICRH antennae, which is $\approx 1.0 \mathrm{~m}$. The speed of its vertical extension is estimated to be $50-100 \mathrm{~m} / \mathrm{s}$.

\section{Poloidal injection angle}

The intersection region of the beam and the resonance cylinder can be moved vertically varying the poloidal launching angle. From JT-60U (O1) [16] and KSTAR (X2) [4] it is reported that it is favorable if this region is located somewhat below the mid-plane. These results are of different nature: In the $\mathrm{O} 1$ case of JT-60U, the initial plasma formation (before $U_{\text {loop }}$ is applied) is hardly affected by the poloidal angle variation, but the plasma current evolution varies. The authors speculate that the initial plasma forms at different locations, but data on the position of the current center are not shown. It also seems possible that the confined plasma forming around a current center (rather fixed in space) is heated with varying efficiency depending on the poloidal angle. In the X2 case of KSTAR significant variations of the delay between onset of ECRH power and $H_{\alpha}$ rise are found in the preionisation phase without $U_{\text {loop }}$, the delay being shorter for beams crossing the resonance cylinder below the midplane. The authors speculate that the $\nabla \vec{B} \times \vec{B}$ drift of the electrons is important, but quantitatively one finds that for $B_{z} R=10^{-3} \mathrm{Tm}$ and $10 \mathrm{eV}$ electron energy, the vertical component of the parallel velocity exceeds the vertical drift velocity if $v_{\|} / v_{\perp}>0.015$. Under these conditions $\left(v_{\perp}>>v_{\|}, E_{k i n}=10 \mathrm{eV}, B_{\Phi} R=5 \mathrm{Tm}\right)$ the $\nabla \vec{B} \times \vec{B}$ drift is $2 \mathrm{~m} / \mathrm{s}$ or $2 \mathrm{~mm} / \mathrm{ms}$. In other words, in the collision-less acceleration phase the drift is dominant but very small. In the collisional rather cold plasma which corresponds to the $H_{\alpha}$ emission $v_{\|}$will be a significant fraction of $v_{\perp}$ and therefore $v_{\|}$dominates the vertical movement of the charged particles. This is also in line with figure 4, in which an up-down symmetry in the vertical plasma extension is observed. One possible explanation for the observation in KSTAR may be related to the position of the launcher below the midplane. A beam crossing the resonance cylinder below the midplane has a smaller vertical inclination and bounces more often back and forth through the plasma in this poloidal cross section. The path of the 

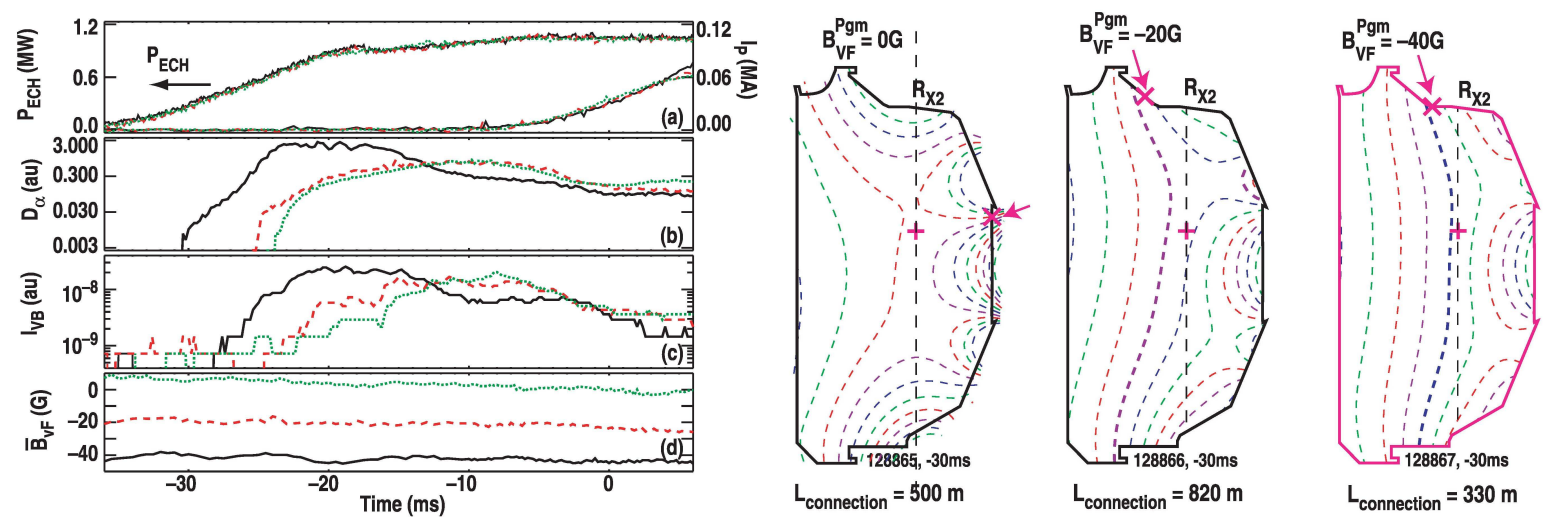

Figure 5. Pre-ionisation in DIII-D, scan of vertical field $B_{z}$ using a slow ECRH power ramp and corresponding poloidal flux surfaces. (From [22], figures 13 and 14, with permission of G.L. Jackson.) The $B_{z}$ values connecting the time traces on the left to the equilibria on the right are given in the lowest box on the left and above the equilibria $\left(B_{V F}^{P g m}\right)$, respectively.

reflected beam significantly varies as the plasma facing structures in KSTAR are completed such that they become more axis-symmetric. It will be interesting to follow in how far the length of the collision-less heating phase is influenced. First results with PFCs upgraded in 2010 are presented in [17].

\section{Prefill pressure}

The values in table 1 show significant inter-machine variation in terms of breakdown field and required ECRH power indicating the influence of additional parameters. In fact also the intramachine variations are of similar relative size. Two major parameters are the prefill pressure and the structure of the poloidal field null, which are addressed in this and the next section.

All devices find that ECRH assist allows to use significantly higher prefill pressures than for optimised low voltage breakdown. Systematic scans have been performed in TS, JT60U, FTU, DIII-D (O1 and X2) and KSTAR. For O1 the possible range of prefill variation spans more than a factor of 4 . For X2 at least a factor of 3 is found in DIII-D and KSTAR. Both experiments find that the upper limit increases with ECRH power. For constant ECRH power, the range for successful breakdown with X2 varies with the injection angle. This is the reason for the initially negative results in DIII-D for X2-breakdown with large toroidal launching angles as explained in [21]. Following the discussion at the end of the section 2 it is most likely that for X2 the pressure-range narrows with increasing toroidal angle as the collisionality condition has to be met more accurately, if the local electric field is reduced by a toroidal inclination of the beam. For O1, FTU finds that $20^{\circ}$ degree inclined injection widens the usable pressure range. 


\section{Field null}

The issue of details of the field null seems to be especially crucial for X2-heating, as found in TS, where $\mathrm{O} 1$ experiments were performed prior to X2 experiments. Also DIII-D, TS, KSTAR and AUG find strong effects, if $P_{E C R H}$ is close to the threshold. Ideally the poloidal field would be zero in the whole vessel during pre-ionisation and the electrons would only drift vertically with the $\nabla B \times B$ drift. In reality, a finite vertical field remains even in the plasma center such that parallel velocities have a non-zero projection in vertical direction (see section 3). Additionally, non-zero radial field components exist as well, such that the corresponding projection of $v_{\|}$can move the electron radially. The 2-D patterns of $B_{R}$ and $B_{z}$ determine the poloidal field-null structure. It determines the connection lengths to the wall, which should be large. As found in DIII-D, it is also beneficial if the projected field lines are vertical in the vicinity of the resonance cylinder, i.e. $B_{z}>>B_{R}$ in this region [22] as shown in figure 5. This makes fast electrons stay longer close to the resonance such that the ECRH beam is absorbed more efficiently. In DIII-D, configurations with the lowest $B_{z}$ have $B_{R} \approx B_{z}$. It can be seen that in this case an additional vertical magnetic field of $\approx 5 \mathrm{mT}$ facilitates pre-ionisation although it reduces the connection length. The value of such an additional field depends on the initial field-null structure. TS and KSTAR find optimum additional $\left|B_{z}\right|<2$ mT. For AUG, two field nulls have been used accidentally. In 2008 a very large null has been used with $B_{p o l}<2 \mathrm{mT}$, which yielded a perfectly vertical initial ECR plasma (fig. 4). In 2009 the area where $B_{p o l}<2 \mathrm{mT}$ was much smaller and the initial ECR plasma was bent away from the resonance cylinder. In the latter case additional $B_{z}$ helped. These AUG results indicate that an additional $B_{z}$ is not always necessary to align the initial plasma with the resonance cylinder, but optimization of the field null may be important too. Following the argument at the end of section 2 on reentry of an electron in the ECR beam after a toroidal turn, the diameter of the ECR beam may also be relevant: the vertical displacement of the field line after one toroidal term depends linearly on $B_{z}$. If the ECR beam is wide, higher $B_{z}$ values may be tolerable than for narrow beams. This could explain why DIII-D with rather wide ECR beams performs well with relatively high $B_{z}$ values.

Some interesting issues related to the pre-ionisation by ECRH are not followed up in this paper, since their relation to the other findings presented here is not clarified yet. Among these are the toroidal currents found in the pre-ionisation phase in most Tokamaks $(>30 \mathrm{kA}$ in recent DIII-D experiments [23]) and the observation in KSTAR that no pre-ionisation occurs with no current in the poloidal field coils (i.e. $B_{\Phi}$ only) [17].

We note here that also for the spherical tokamak QUEST, ECRH assisted breakdown is reported with non-zero vertical field. Since the resonance is located in this case essentially on the inner heat shield, it is not clear that the process is directly comparable to the other Tokamaks where the initial plasma generation is well separated from the walls. 

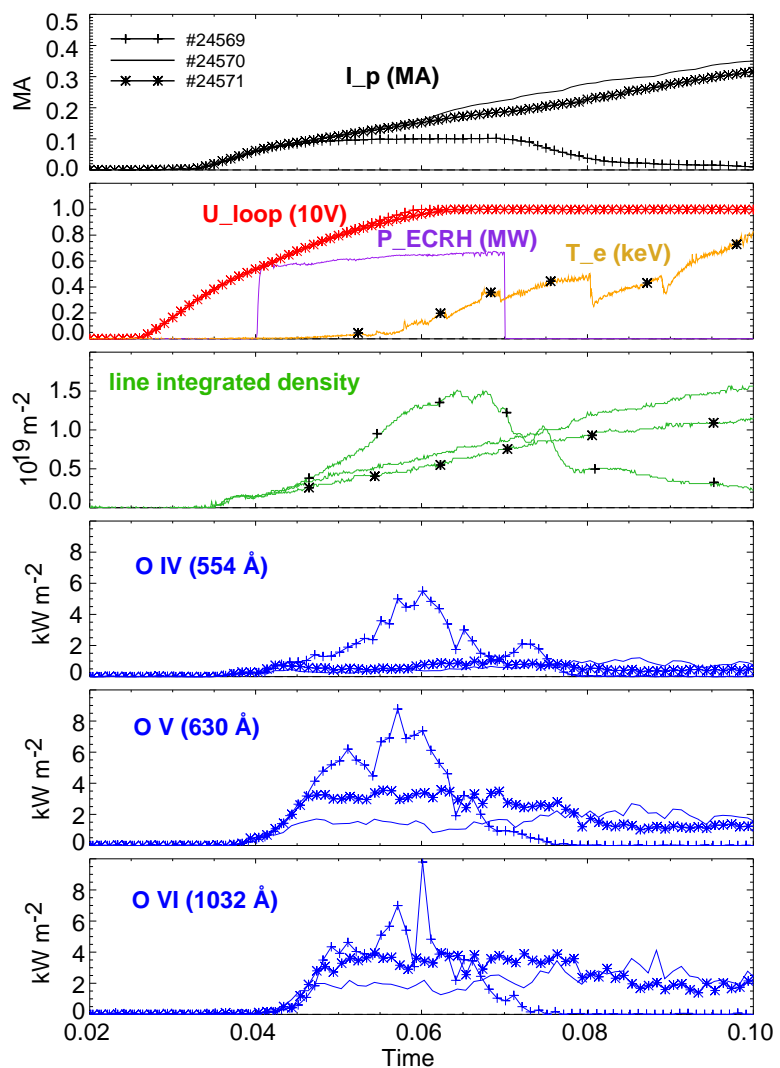

Figure 6. Sequence of successive plasma start-ups on AUG. \#24569 failed to sustain breakdown (time traces with crosses), in \#24570 ECRH was applied (time traces without symbols) and in \#24571 breakdown was achieved without ECRH due to conditioning effects of the previous discharge (time traces with stars). Line averaged density is measured perpendicular in the midplane, as is the radiation from partially ionised oxygen, for which a SPRED VUV spectrometer is used. Other parameters such as field null and pre-fill were kept constant.

\section{Current formation and impurity burn-through}

As the loop voltage is applied in Tokamaks, a toroidal current rises, which may lead to the formation of closed flux surfaces. Initially, it has to develop in a location determined by feed-forward(FF) control of the poloidal field coils, where it can grow until it is large enough that its position can be determined by the magnetics and feed-back control can take over. In the phase of FF-control the value of $B_{z}$ is also crucial. Since $\dot{B}_{z}$ is limited, the values of $B_{z}$ during pre-ionisation and during current formation are strongly coupled and it is hard to say which one determines the optimum. It is usually found that the current does not form at the ECRH resonance. On AUG with ECRH the current forms close to the outboard limiters but without ECRH it forms close to the inner heat shield although poloidal field currents are programmed to be identical and the resonance is closer to the inboard side. The difference may be due to different vessel currents, since the loop voltage is different in both cases. These vessel currents are hard to determine, which prevented so far an inter-machine comparison. In fact, in DIII-D the current forms on the in-board side in both cases and it would be definitely 
interesting to understand the differences between both devices. As mentioned above, during the ionisation of light impurities too much power may be radiated that the breakdown is not sustained. Obviously this radiation is related to the impurity densities, such that machine condition plays an important role for this phase. In AUG it has been found that the current ramp-rate is significantly higher in the boronised machine as compared to the unboronised $\mathrm{W}$-coated machine. The effect is most likely due to the remotely deposited Boron which acts as a getter for light impurities. On AUG and DIII-D, ECRH has also been used successfully after ohmic breakdown to burn through this radiation barrier. For AUG this is illustrated with a sequence of three successive discharges in figure 6. The first discharge had a non-sustained breakdown, in the second discharge ECRH was applied from $15 \mathrm{~ms}$ until $45 \mathrm{~ms}$ after start of the $\mathrm{U}_{\text {loop }}$-ramp and the third discharge successfully broke down without ECRH assist. It is clearly seen that the radiation due to incompletely ionised oxygen is significantly reduced with ECRH even in comparison to the third discharge proving that this is not only a conditioning effect. This means that with ECRH the electron temperature rises quicker. Unfortunately a direct proof using ECE emission is missing, since the ECE system on AUG was not used with early ECRH, not to expose the diagnostic to excessive stray radiation. We note that KSTAR has recently changed ECRH assist from a pre-ionisation scheme to a scheme sustaining ohmic breakdown in order to improve reproducibility and to reduce impurity production due to the strong interaction of the non-absorbed beam with in-vessel components during the first milliseconds in the pre-ionisation sequence [17]. This new sequence has been run with X2- and O1-heating using $110 \mathrm{GHz}$ at different toroidal field values.

\section{Minimum power and size scaling}

Usually it is found that more ECRH power widens the operational range for breakdown for essentially all relevant parameters. Inversely, this means that all parameters have to be adjusted simultaneously to find the absolute minimum of ECRH power for a specific loop voltage and even when doing so, the result will depend severely on wall conditions. Some partial parameter scans were done, but no machine has systematically tried to reduce the required ECRH power to the bare minimum. Especially for the field null this is a high dimensional minimisation procedure. Still, it is noted that TS and JT-60U which differ a factor of 2 in minor radius find approximately the same values of required $\mathrm{ERCH}$ power $(<0.4 \mathrm{MW})$ to obtain breakdown with $\mathrm{O} 1$ for a toroidal $\vec{E}$-field of $0.25 \mathrm{~V} / \mathrm{m}$. It is also noted that KSTAR, driven by limited ECRH power in the early stage of the machine, found a parameter range for $\mathrm{X} 2$ assisted plasma breakdown, with ECRH power well below values found on DIII-D and AUG, indicating some margin in the results presented here. It is also noted that the machines using X2 assist in these joint experiments are of similar size, such that no conclusions on a size scaling can be drawn. 

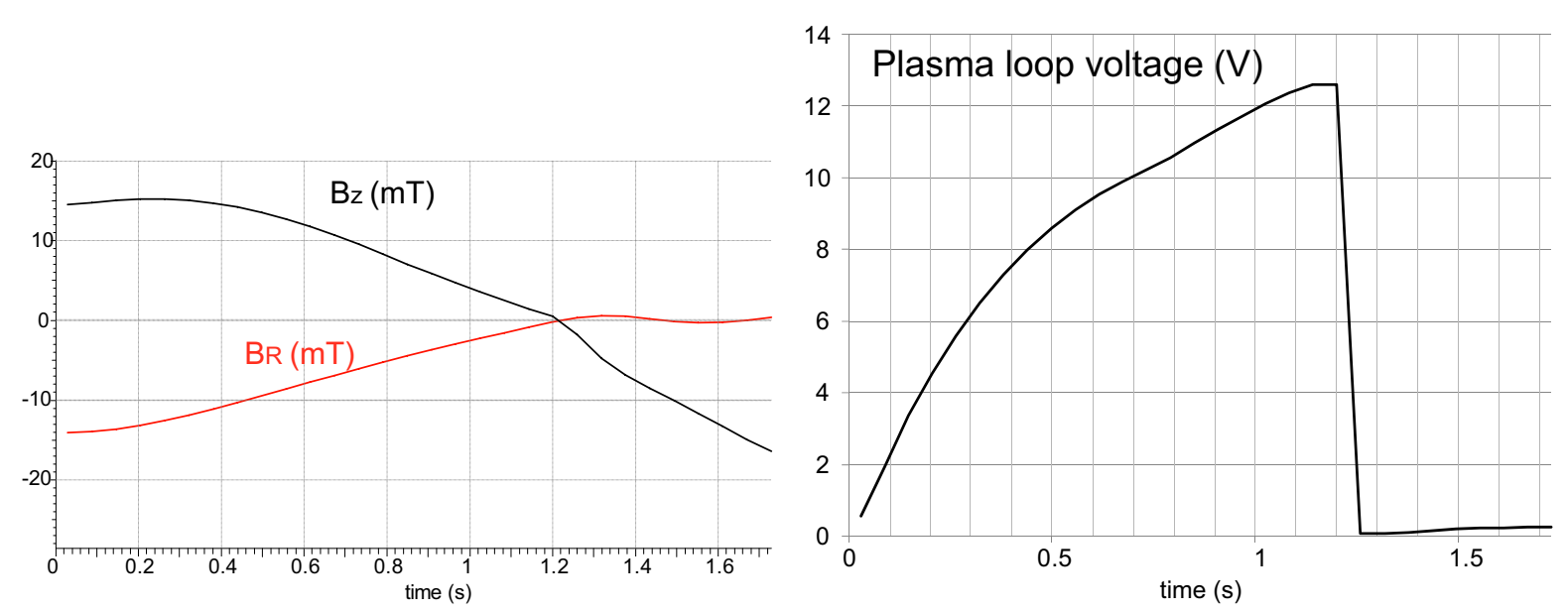

Figure 7. Estimated wave forms for $B_{z}, B_{R}$ and $U_{\text {loop }}$ during breakdown in ITER in the center of the breakdown region for a scenario with full field, fully charged central solenoid and initial current formation on low field side. From [24], figures 6-5, 6-6 and 6-13. Breakdown occurs at $1.2 \mathrm{~s}$.

\section{Consequences for ITER}

In summary it is found that the toroidal inclination of the beams poses no principal problem to ECRH assisted breakdown, but the required ECRH power may be higher up to a factor of two. Unfortunately it is difficult to transfer the results on ECRH assist directly to ITER, since in ITER the field null is only formed when a significant fraction of the loop voltage is already applied [24], because the vessel screening times are large (see figure 7). This is in contrast to most of the performed experiments for which the loop voltage initially was virtually zero, in order to separate the pre-ionisation phase from the current formation phase. This is the case for all X2 and most O1 experiments, except the experiments in TS and FTU where breakdown schemes were run, which use $\mathrm{O} 1$ in a similar timing-scheme as planned for ITER at full field.

The results from FTU experiments, which use ECRH only after application of the loop voltage are shown in figure 8. With ECRH, breakdown is possible at significantly lower loop voltage. The delay of current and temperature rise with respect to the start of the ECRH may be due to the time evolution of the field null, which moves radially outward toward the ECRH resonance. This effect which has a strong similarity to the ITER plans will be analysed in future experiments at FTU. In TS, the loop voltage and a good field null are applied before the ECRH. In that case (fig. 3) the ECRH triggers plasma breakdown and current rise. These results indicate that $\mathrm{O} 1 \mathrm{ECRH}$ assist is expected to reduce the necessary breakdown voltage at full field in ITER. The results in TS and JT-60U which required significantly less than $1 \mathrm{MW}$ of ECRH power suggest that a small fraction of the ITER ECRH power should be sufficient for this purpose.

Using the ITER breakdown-timing at half magnetic field (X2), the reduction of the breakdown voltage by ECRH assist may be significantly smaller, since ionisation with X2 requires that the electrons stay long enough in regions with high ECRH field, from which 


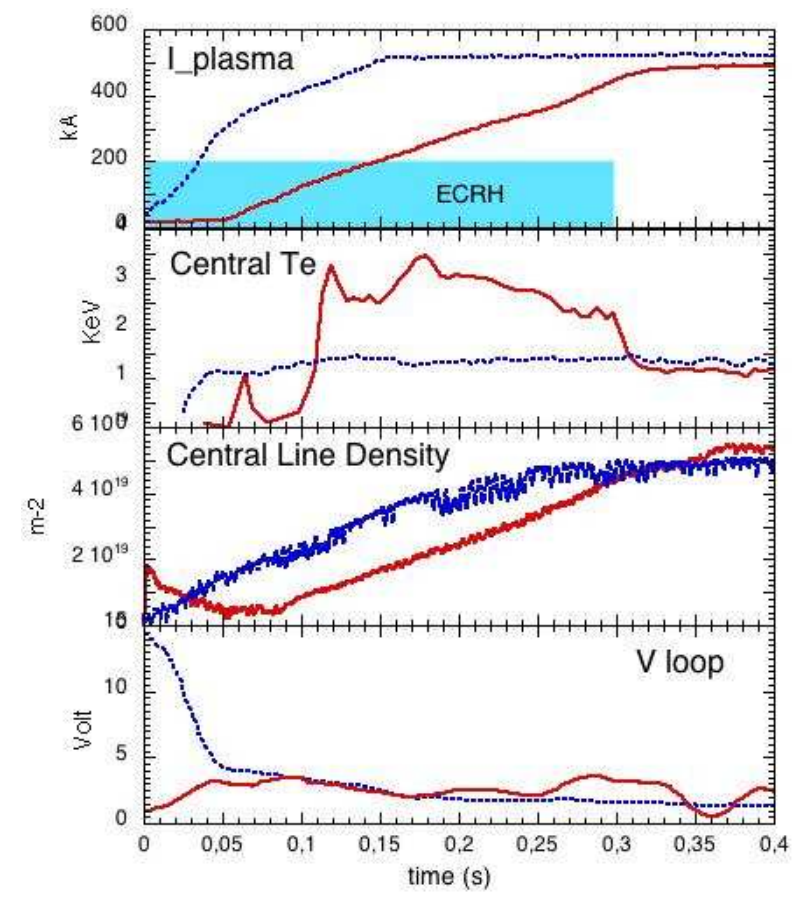

Figure 8. Breakdown in FTU ohmic (blue) and with ECRH assist (red). Shown are plasma current, central $T_{e}$, central line averaged density and loop voltage. The blue shaded area represents the wave form of the ECRH power $(400 \mathrm{~kW})$ in case of ECRH assist.

they may be drawn away by the non-zero loop voltage before taking up sufficient energy from the wave-field. This issue has been recognized and will be addressed in the framework of a joint ITPA-IOS experiment, also using at KSTAR a new $>0.8 \mathrm{~kW}, 170 \mathrm{GHz}$ gyrotron [17], a variant of the Japanese ITER gyrotron [25].

It is important to understand that the difference between $\mathrm{O} 1$ and $\mathrm{X} 2$ discussed above refers only to the initial breakdown. As pointed out in section 6 both schemes strongly enhance impurity ionisation if applied during the current formation phase. Also in ITER both schemes have the potential to convert an unsustained ohmic breakdown into a sustained ECRH assisted breakdown for otherwise unchanged parameters.

\section{Disclaimer}

As for all publications with co-authors from the ITER Organization, the latter requires to add the following disclaimer: "The views and opinions expressed herein do not necessarily reflect those of the ITER Organization."

\section{References}

[1] SIPS, A. C. C. et al., Nucl. Fusion 49 (2009) 085015.

[2] LLOYD, B. et al., Nucl. Fusion 31 (1991) 2031.

[3] BUCALOSSI, J. et al., in Fusion Energy 2008 (Proc. 22nd Int. Conf. Geneva, 2008) CD-ROM 
and http://www-naweb.iaea.org/napc/physics/FEC/FEC2008/html/index.htm, pages EX/P6-12, Vienna, IAEA.

[4] BAE, Y. S. et al., Nucl. Fusion 49 (2009) 022001.

[5] BAE, Y. S., 2010, NFRI, Daejeon, Korea, private communication.

[6] LLOYD, B. et al., Plasma Phys. Controlled Fusion 38 (1996) 1627.

[7] OMORI, T. et al., Fusion Eng. Des. 86 (2011) in press, available online.

[8] JACKSON, G. L. et al., Nucl. Fusion 47 (2007) 257.

[9] NAGASAKI, K. et al., Nucl. Fusion 45 (2005) 13.

[10] JACKSON, G. L. et al., Nucl. Fusion 49 (2009) 115027.

[11] KESSEL, C. E. et al., Nucl. Fusion 49 (2009) 085034.

[12] VOITSEKHOVITCH, I. et al., Plasma Phys. Controlled Fusion 52 (2010) 105011.

[13] IMBEAUX, F. et al., in Fusion Energy 2010 (Proc. 22nd Int. Conf. Daejeon, 2010) CD-ROM and http://www-naweb.iaea.org/napc/physics/FEC/FEC2010/html/index.htm, pages ITR/P1-20.

[14] JACKSON, G. L. et al., Phys. Plasmas 17 (2010) 056116.

[15] GRANUCCI, G. et al., Plasma start-up results with EC assisted breakdown on FTU, submitted to Nucl. Fusion (January 2011).

[16] KAJIWARA, K. et al., Nucl. Fusion 45 (2005) 694.

[17] JOUNG, M. et al., in Fusion Energy 2010 (Proc. 22nd Int. Conf. Daejeon, 2010) CD-ROM and http://wwwnaweb.iaea.org/napc/physics/FEC/FEC2010/html/index.htm, pages EXW/P2-05.

[18] BUCALOSSI, J. et al., Nucl. Fusion 48 (2008) 054005.

[19] KIRNEVA, N. A. et al., Plasma start-up optimisation with 2nd harmonic ECR pre-ionization in T-10 tokamak, in Europhysics Conference Abstracts (CD-ROM, Proc. of the 34th EPS Conference on Plasma Physics, Warsaw, 2007), edited by GASIOR, P. and WOLOWSKI, J., volume 31F, pages P-1.164, Geneva, 2007, EPS.

[20] KASPAREK, W. et al., Int. J. Infrared Millimeter Waves 22 (2001) 1695.

[21] JACKSON, G. L. et al., Experiments simulating ITER Rampdown and startup scenarios in the DIII-D tokamak, in Europhysics Conference Abstracts (CD-ROM, Proc. of the 36th EPS Conference on Plasma Physics, Sofia, 2009), edited by MATEEV, M. and BENOVA, E., volume 33E, pages P-4.139, Geneva, 2009, EPS.

[22] JACKSON, G. L. et al., Fusion Sci. Tech. 57 (2010) 27.

[23] JACKSON, G. L. et al., in Fusion Energy 2010 (Proc. 22nd Int. Conf. Daejeon, 2010) CD-ROM and http://www-naweb.iaea.org/napc/physics/FEC/FEC2010/html/index.htm, pages EXS/P2-11.

[24] KAVIN, A. A. et al., Study of Plasma Start up, ITER Contract No. ITER/CT/09/430000003, 2nd Intermediate Report, Study of plasma initiation using TRANSMAK code: ITER design "baseline 2010”, Technical Report ITER, private communication, 2010.

[25] SAKAMOTO, K. et al., in Fusion Energy 2010 (Proc. 22nd Int. Conf. Daejeon, 2010) CD-ROM and http://www-naweb.iaea.org/napc/physics/FEC/FEC2010/html/index.htm, pages ITR/2-5Rb. 